\title{
A 60 pc counter-rotating core in NGC $4621^{\star}$
}

\author{
F. Wernli ${ }^{1}$, E. Emsellem ${ }^{1}$, and Y. Copin ${ }^{2}$ \\ 1 Centre de Recherche Astronomique de Lyon, 9 Av. Charles André, 69561 Saint-Genis Laval Cedex, France \\ ${ }^{2}$ Institut de physique nucléaire de Lyon, 4 rue Enrico Fermi, 69622 Villeurbanne Cedex, France
}

Received 28 March 2002 / Accepted 10 September 2002

\begin{abstract}
We present adaptive optics assisted OASIS integral field spectrography of the S0 galaxy NGC 4621. Two-dimensional stellar kinematical maps (mean velocity and dispersion) reveal the presence of a $\sim 60 \mathrm{pc}$ diameter counter-rotating core (CRC), the smallest observed to date. The OASIS data also suggests that the kinematic center of the CRC is slightly offset from the center of the outer isophotes. This seems to be confirmed by archival HST/STIS data. We also present the HST/WFPC2 $V-I$ colour map, which exhibits a central elongated red structure, also slightly off-centered in the same direction as the kinematic centre. We then construct an axisymmetric model of NGC 4621: the two-integral distribution function is derived using the Multi-Gaussian Expansion and the Hunter \& Qian (1993) formalisms. Although the stellar velocities are reasonably fitted, including the region of the counter-rotating core, significant discrepancies between the model and the observations demonstrate the need for a more general model (e.g. a three-integral model).
\end{abstract}

Key words. galaxies: elliptical and lenticular, cD - galaxies: evolution - galaxies: individual: NGC 4621 - galaxies: formation galaxies: kinematics and dynamics - galaxies: nuclei

\section{Introduction}

The fact that early-type galaxies are not necessarily oblate major-axis rotators has been well known since the end of the 1970s. The number of objects showing significant minoraxis rotation attained ten in 1988 (Wagner et al. 1988), and some of these objects later showed evidence for kinematically decoupled core structures (NGC 4365, NGC 4406: Bender 1988; NGC 4589: Moellenhoff \& Bender 1989; NGC 5982: Wagner 1990). The most cited formation scenario for such structures is the hierarchical model, which involves single or multiple merging steps (see e.g. Cole et al. 1994 and references therein). Observations of kinematically decoupled cores can therefore be used to constrain the merger tree of galaxies. However the total fraction of early-type galaxies containing such a core is poorly known (de Zeeuw \& Franx 1991). Such cores are generally revealed using long-slit measurements with $1^{\prime \prime} F W H M$ or worse spatial resolution: this introduces an a priori assumption on the geometry of the central structures, and prevents the detection of cores with small apparent sizes. Finally, the rough characteristics of these cores (fraction in mass, relative age and metallicity) remain very uncertain, as very few studies exist and are often limited to morphological data (see Carollo et al. 1997 for 15 KDCs).

Send offprint requests to: $\mathrm{F}$. Wernli, e-mail: fabien@obs.univ-lyon $1 . f r$

* Based on observations collected at Canada-France-Hawaii Telescope, which is operated by CNRS of France, NRC of Canada, and the University of Hawaii.
Recent studies (e.g. Verolme et al. 2002) show that state-ofthe-art models combined with two-dimensional integral-field spectroscopic data are required to constrain precisely the global physical parameters of early-type galaxies, such as the inclination angle or the mass-to-light ratio, as well as the central characteristics for objects exhibiting features such as kinematically decoupled cores (e.g. the core mass of IC 1459, Cappellari et al. 2002). Moreover, integral-field spectroscopy also provides access to line-strength maps: the detailed kinematic information can thus be coupled to the line indices in order to more easily disentangle the core from the host galaxy (Davies et al. 2001).

The SAURON survey (Bacon et al. 2001a; de Zeeuw et al. 2002) was carried out in order to study the integral field kinematics of a sample of 72 early-type galaxies over a wide field of view $\left(>33^{\prime \prime} \times 41^{\prime \prime}\right)$. In parallel, to obtain complementary high spatial resolution data, we designed a program to observe their central parts $\left(\sim 5^{\prime \prime} \times 5^{\prime \prime}\right)$ with the integral field spectrograph OASIS (Bacon et al. 2000). The aim is to probe the central spatial morphology and dynamics of a subset of galaxies in the SAURON sample, in order to link them with the global properties of the host galaxies provided by SAURON and other wide field studies. The additional use of PUE0 (Rigaut et al. 1998), the Canada France Hawaii Telescope AO bonnette, with OASIS allows us to reach resolutions of about $0.25(\sim 12 \mathrm{pc}$ at $10 \mathrm{Mpc})$ in the red part of the visible when bright guiding sources are available.

NGC 4621, an S0 galaxy (Lauer et al. 1995), with $M_{V}=9.6$, located in the Virgo cluster $(D=18.3 \mathrm{Mpc}$, 
Tonry et al. 2001), is the first target of the subsample that was observed with OASIS/PUEO. Its steep power-law central luminosity profile ( $\gamma=2.03$, Gebhardt et al. 1996) is ideally adapted to serve as a guiding source for the AO. NGC 4621 is close to edge-on, and its innermost $6^{\prime \prime}$ isodensities reveal a nuclear disk (Sil'Chenko et al. 1997).

In this paper, we present the first results of our OASIS observations of NGC 4621, with the discovery of the smallest counter-rotating stellar core observed to date. The scale of the core is $60 \mathrm{pc}$, while the previously detected cores have an average radius of around $1 \mathrm{kpc}$ (see the sample of Carollo et al. 1997). Detailed information on the different data sets used in this work, including HST/WFPC2 and STIS observations, are provided in Sect. 2. The corresponding measured photometry and stellar kinematics are presented in Sect. 3. We computed a two-integral distribution function (DF) model of the galaxy, which is presented in Sect. 4. The discussion is carried out in Sect. 5.

\section{Observational data sets}

\subsection{OASIS data}

\subsubsection{Observations}

Ca-triplet OASIS exposures of NGC 4621 were obtained in January 2000 using the medium spectral configuration (MR3: 8346-9152 $\AA$ ) at the f/20 (PUE0) focus of the CFHT, and a spatial sampling of 0 .' $^{\prime} 16$. Eight out of ten $30 \mathrm{~min}$ exposures were fully reduced using the dedicated XOasis package ${ }^{1}$, and merged into a single frame (two frames were discarded due to bad seeing conditions and associated loss of guiding). The resulting 3D dataset ${ }^{2}$ probes about $4^{\prime \prime} \times 4^{\prime \prime}\left(300 \times 300 \mathrm{pc}^{2}\right)$, with a resolution of $\sigma=70 \mathrm{~km} \mathrm{~s}^{-1}$. We modeled the PSF of the merged datacube using the sum of two concentric Gaussians: their parameters were retrieved (via a least-square procedure) by comparison with the WFPC2/HST F814W frame as in Bacon et al. (2001b). The resulting FWHM of the merged datacube is 0.51 .

The total $\mathrm{S} / \mathrm{N}$ (summed over the bandwidth) drops very quickly towards the outer parts, as it is 65 at the center, 20 at $1^{\prime \prime}$ and 10 at $2^{\prime \prime}$ on the major-axis. We thus convolved the datacube with a Gaussian of variable width in order to obtain a higher $S / N$ over the field (see Fig. 1).

\subsubsection{Kinematic measurements}

We used a tuned version of the Fourier Correlation Quotient (FCQ; Bender 1990) method to derive the line of sight velocity distributions (LOSVD) at each measured spatial position. We used a single template star (G8III: HD073665), but tested the stability of the kinematical mesurements with different templates, and different continuum subtraction parameters, only resulting in minor differences. The LOSVDs were then fitted using a single Gaussian function, yielding the mean velocity and dispersion maps. We could not obtain reasonable maps of

\footnotetext{
${ }^{1}$ http://wWW-obs.univ-lyon1.fr/ ${ }^{\circ}$ oasis/home/index.html

${ }^{2}(\alpha, \delta, \lambda)$, one spectrum per spatial element
}

higher order moments, due to the rather low signal to noise ratio (even after the convolution performed on the datacube). The error bars on the velocity and dispersion measurements were computed via a Monte-Carlo algorithm using the measured $S / N$ (see Copin 2000, Ph.D. Thesis).

\subsection{Ancillary data}

\subsubsection{Photometry}

In order to obtain an accurate three-dimensional mass model of the galaxy we need both wide-field and high-resolution photometry to probe the visible mass up to large radii and to correctly sample the central structure (the cusp). We used a $20^{\prime}$ $V$ band image of the galaxy taken at the OHP $2 \mathrm{~m}$ Telescope, kindly provided by R. Michard (Idiart et al. 2002). The central regions were examined with the help of WFPC2/HST data $^{3}$ retrieved from the archive (F555W and F814W filters, Faber et al. 1997, \#5512). For both bands, three unsaturated frames were merged and cosmic ray-corrected (330 s and $230 \mathrm{~s}$ exposure time for F555W and F814W filters respectively). We performed photometric calibration using the VEGAMAG standard, and used the PSFs computed using tinytim ${ }^{4}$. We adjusted the levels of the OHP image to the F555W frame taking into account the different spectral domains and the PSF.

\subsubsection{Long-slit kinematics}

We used long slit data from Bender et al. (1994, hereafter BSG94; data kindly provided by R. Bender) in order to complement the OASIS data up to large radii: major and minor axis velocity and dispersion measurements are available inside $40^{\prime \prime}(3.5 \mathrm{kpc})$, with a seeing of $\sim 11^{\prime \prime} 8$ FWHM (other kinematical - and line-strength - measurements were published by Sil'chenko 1997, but with a lower spatial resolution).

We finally reduced unpublished HST/STIS major-axis data (Green, ID \#8018; G750M grating, Ca-Triplet) in order to examine the central kinematics of NGC 4621 at high spatial resolution. Two exposures with a total of $72 \mathrm{~min}$ were available. The calibrated data were retrieved via the STScI data archival system (calstis pipeline). Appropriate flat field exposures were retrieved to correct for the fringing (mkfringeflat/defringe IRAF routines, Goudfrooij \& Christensen 1998), critical at these wavelengths, as well as five K0-III kinematical template star exposures (HR7615, Green, \#7566). Further rejection of cosmic rays was then performed on the NGC 4621 individual exposures, before recentring and merging. The stellar kinematics were extracted using the same FCQ and fitting methods as for the OASIS data. As for the OASIS datacubes, the $S / N$ of the STIS data was found sufficient only to derive the mean stellar velocity and dispersion within the central arcsecond.

\footnotetext{
${ }^{3}$ Based on observations collected with the NASA/ESA HST, obtained at STScI, which is operated by AURA, Inc, under NASA contract NAS5-26555.

${ }^{4}$ http://www.stsci.edu/software/tinytim/tinytim.html
} 

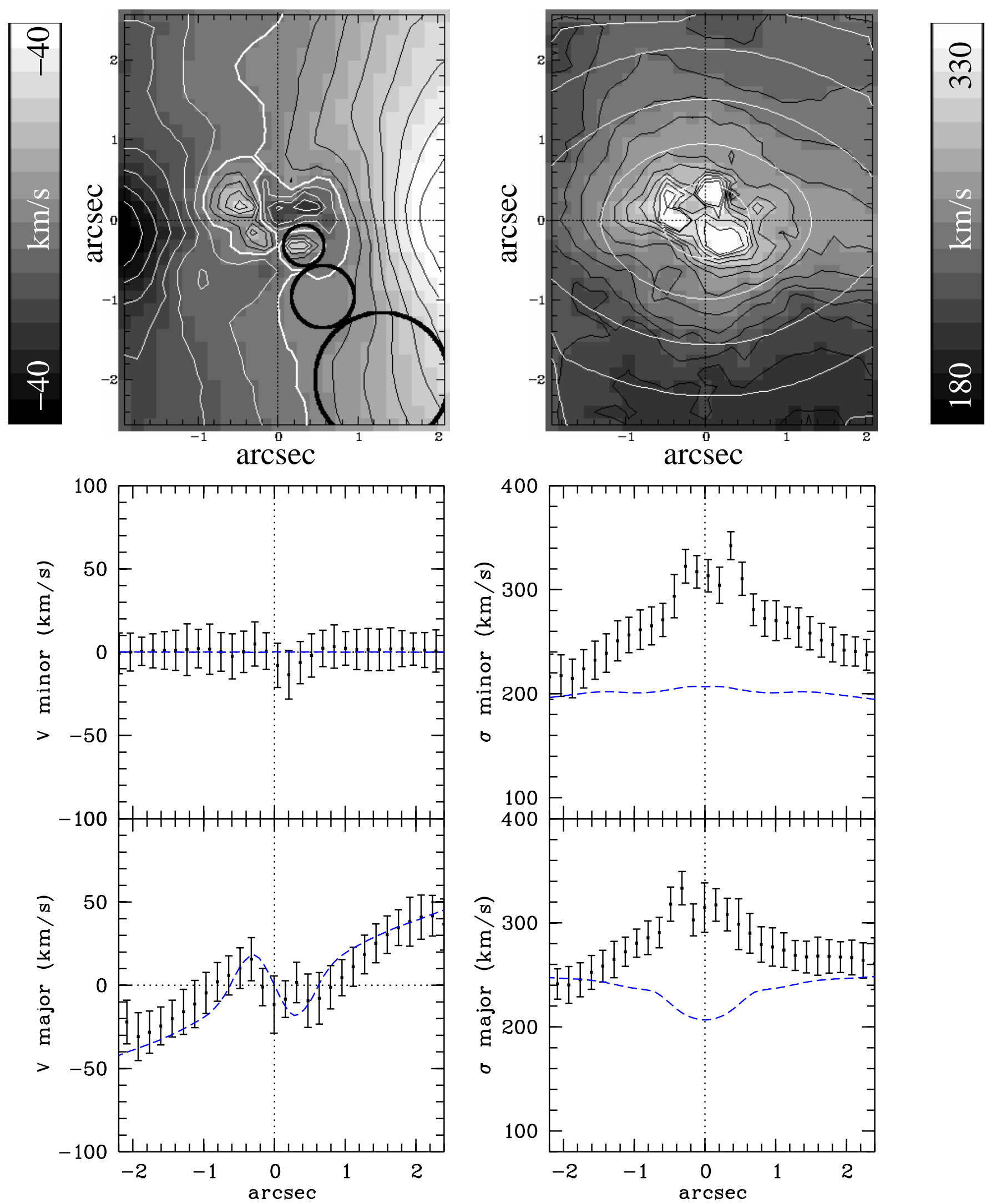

Fig. 1. OASIS stellar kinematic data of NGC 4621. Top left: mean velocity field. The zero-velocity curve has been emphasized with a white thick line. Top right: velocity dispersion. White contours are reconstructed isophotes. The data have been smoothed to improve the signal to noise: the black circles represent the beam sizes $(F W H M)$. Below the cuts along the major and the minor axes are shown along with the best fitting two-integral model (dashed lines, see Sect. 4). 

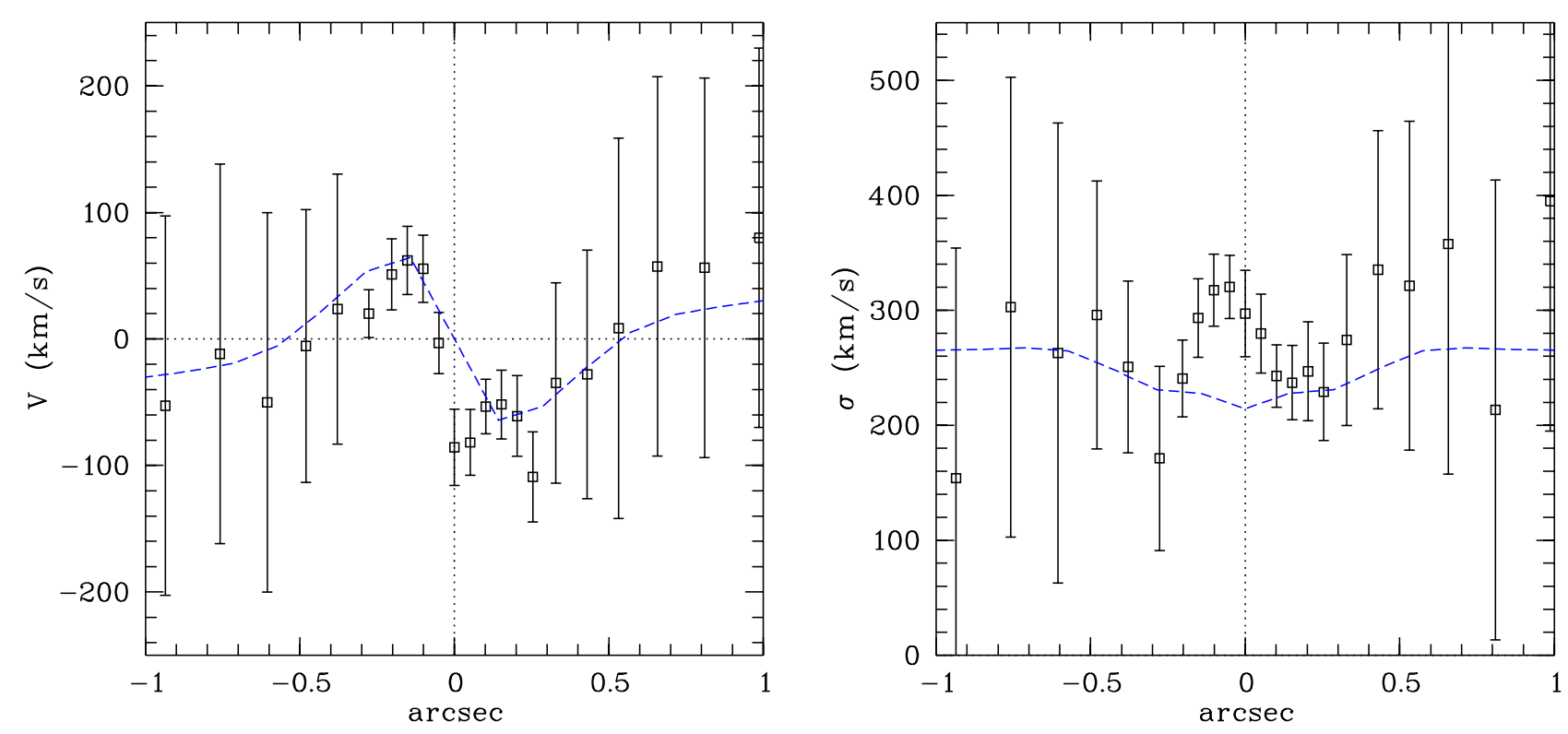

Fig. 2. STIS mean velocity (left) and dispersion (right) profiles along the major axis. The dashed line corresponds to the best fitting two-integral model (see Sect. 4). The velocity dispersion is clearly underestimated by the present model, which hints for the need of an additional central dark mass, a more general treatment (three-integral model) or both.

\section{Results}

To avoid any confusion due to the complexity in both the photometry and the kinematics of NGC 4621, we will use the following convention: the photometric major axis of the galaxy (as measured by HST) defines the $x$-axis, the negative values being in the SE quadrant, referred to as the left side of the galaxy. The center $(0,0)$ is defined as the center of the HST isophotes of NGC 4621 within $1^{\prime \prime}$, excluding the central $0{ }^{\prime} 1$, the isophotes of which exhibit a significant asymmetry (see next section). The centering and rotation procedures of the HST frames have been performed using an algorithm which minimizes the standard deviation of the recentered and rotated frame subtracted by its flipped counterpart. This leads to accuracies of 0.1 degrees and 0 ' $^{\prime} 005$. All figures in this paper share a common orientation, as shown in Fig. 3.

\subsection{Stellar velocity and velocity dispersion}

The OASIS stellar mean velocity and dispersion maps are presented in Fig. 1. The datacubes have been centered and rotated in order to match the HST data, using the center and angle provided by the PSF fitting procedure mentioned in Sect. 2. The velocity field reveals a clear counter-rotating core (CRC). The position of the zero-velocity curve is used to measure the center and the size ${ }^{5}$ of the CRC: it is $1^{\prime \prime} .7(150 \mathrm{pc})$ in diameter, and off-centered by $\sim 0{ }^{\prime} .2$ towards the SE. The total velocity amplitude observed within the central $1^{\prime \prime}$ along the major axis is $100 \mathrm{~km} \mathrm{~s}^{-1}$, while the peak-to-peak velocity amplitude of the CRC's is only $35 \mathrm{~km} \mathrm{~s}^{-1}$. The stellar velocity dispersion map peaks at $\sim 330 \pm 25 \mathrm{~km} \mathrm{~s}^{-1}$. The dispersion map however exhibits high frequency substructures in the central 0.'5 (Fig. 1), the minor-axis dispersion profile even having a local minimum

\footnotetext{
5 The limits of the CRC are defined by the zero-velocity curve.
}

at the centre. Considering the amplitude of these structures and their spatial scales (comparable to the local FWHM), we should wait for data with better signal to noise ratio to discuss these features.

The BSG94 data show no significant minor-axis rotation, and a maximum velocity of $140 \mathrm{~km} \mathrm{~s}^{-1}$ at $30^{\prime \prime}$ (see BSG94 or Fig. 7). Besides a relatively weak velocity amplitude in the innermost $4^{\prime \prime}\left(<60 \mathrm{~km} \mathrm{~s}^{-1}\right)$, these data do not show any hint for the existence of the counter-rotating core. We will use the BSG94 kinematics to constrain the dynamical model outside the OASIS field of view.

The STIS velocities and dispersions are shown in Fig. 2. In these data the size of the CRC is 0.7 ( $\sim 60 \mathrm{pc})$ and its velocity center is located at $x=-00^{\prime} 05(\sim 4.5 \mathrm{pc})$. We have carefully checked the centering of the STIS data with respect to the reference WFPC2 images, and found that this offset is robust. At STIS resolution the major-axis velocities of the CRC reach $\sim 75 \mathrm{~km} \mathrm{~s}^{-1}$ with respect to the systemic. The maximum velocity dispersion is $320 \pm 27 \mathrm{~km} \mathrm{~s}^{-1}$, at $x=0$. $^{\prime} 05$. These values are consistent with the OASIS data considering the higher spatial resolution of the STIS data.

\subsection{HST photometry}

Both F555W and F814W images reveal a peculiar structure in the core. Indeed, the luminosity peak is offset from the center of the outer isophotes by 0 .' $^{\prime} 01$ towards the east. The photometric peak is located in the upper left quadrant (Fig. 3). This feature does not seem to be an artefact, as it is clearly visible on each individual HST frame, and in both bands, and cannot be attributed to centering uncertainties (0.005).

The two HST frames were then PSF-crossconvolved and divided to obtain a $V-I$ colour image (Fig. 4). It reveals a central gradient $(V-I$ increases towards the center) with a $y$-axis 


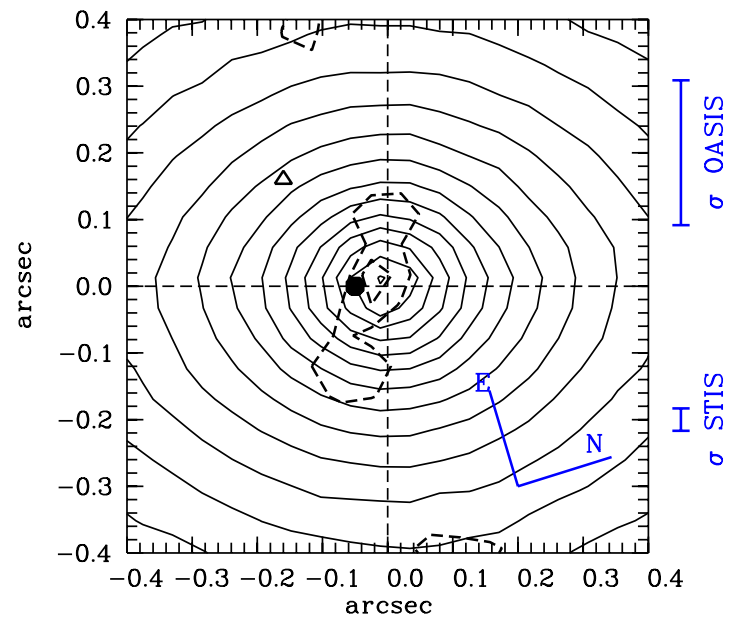

Fig. 3. Inner isophotes of NGC 4621 (WFPC2 F555W, solid contours, step of $0.2 \mathrm{mag})$. The center $(0,0)$ of the galaxy is defined as the center of the outer isophotes (outside $0{ }^{\prime} 1$ ). The open triangle and filled circle correspond to the center of the counter-rotating core (CRC) as measured in the OASIS and STIS data repectively (see Sect. 3.1). The vertical bars correspond to the OASIS and STIS spatial resolutions $(\sigma)$. The dashed contours represent the $V-I$ colour map from WFPC2: levels 1.36 and 1.37 mag. North is 73 degrees clockwise from the vertical axis.

elongated structure centered at $\left(-0 .{ }^{\prime} 02,0{ }^{\prime} 01\right)$ (see dashed line on Fig. 3). The isochromes have slightly higher ellipticities than the isophotes $(\varepsilon=0.5$ at 2 '. 5 for $V-I$, vs. 0.4 for the $V$ frame).

\section{Models}

In this Section, we present the methods used to model the photometry and kinematics of the central region of NGC 4621. The available data suggest that the very central region $(100 \mathrm{pc})$ of NGC 4621 slightly departs from axisymmetry. The CRC seems off-centered in both the OASIS and STIS data. However, the off-centering is only $\sim 4 \mathrm{pc}$. At the resolution of the BSG94 data, this is obviously not resolved. Moreover, the position angle measured in the OHP photometry does not vary more than 2 degrees within $15^{\prime}$. Axisymmetry is therefore a reasonable approximation for a first modelling. Axisymmetric, two-integral models then have the advantage to be semianalytical. We first derived simple Jeans models to roughly constrain the input parameters (inclination, mass-to-light ratio). We then used the Hunter \& Qian (1993) formalism to compute the two-integral distribution function $f$ of NGC 4621, as a function of energy $(E)$ and of the vertical component of angular momentum $\left(L_{z}\right)$. The present modelling is only intended to provide a first view at the dynamics of NGC 4621, so we decided not to include a central dark component: this issue will be examined in detail in a forthcoming paper.

\subsection{MGE}

The first step in modelling NGC 4621 was to build a luminosity model which properly reproduced the observed photometry. We used the Multi Gaussian Expansion (MGE) formalism
Table 1. MGE photometric model of NGC 4621 in the $V$ band. The flattened inner disc components are emphasized in bold.

\begin{tabular}{rrrr}
\hline \hline & $I\left(L_{\odot} \mathrm{pc}^{-2}\right)$ & $\sigma\left(^{\prime \prime}\right)$ & $q$ \\
\hline 1 & $4.502 \times 10^{5}$ & 0.040 & 0.860 \\
2 & $7.713 \times 10^{4}$ & 0.112 & 0.610 \\
3 & $4.792 \times 10^{4}$ & 0.201 & 0.941 \\
4 & $1.161 \times 10^{4}$ & 0.438 & $\mathbf{0 . 3 4 4}$ \\
5 & $1.475 \times 10^{4}$ & 0.516 & 0.919 \\
6 & $4.638 \times 10^{3}$ & 1.036 & $\mathbf{0 . 3 2 5}$ \\
7 & $6.317 \times 10^{3}$ & 1.280 & 0.872 \\
8 & $3.357 \times 10^{3}$ & 2.486 & $\mathbf{0 . 2 7 5}$ \\
9 & $2.700 \times 10^{3}$ & 3.211 & 0.658 \\
10 & $1.326 \times 10^{3}$ & 5.698 & 0.817 \\
11 & $6.313 \times 10^{2}$ & 6.926 & $\mathbf{0 . 3 7 7}$ \\
12 & $6.417 \times 10^{2}$ & 12.468 & 0.639 \\
13 & $3.295 \times 10^{2}$ & 25.674 & 0.627 \\
14 & $8.208 \times 10^{1}$ & 57.091 & 0.633 \\
15 & $1.517 \times 10^{1}$ & 128.782 & 1.000 \\
\hline
\end{tabular}

proposed by Monnet et al. (1992) and Emsellem et al. (1994), which expresses the surface brightness as a sum of twodimensional Gaussians. Assuming the spatial luminosity is also a sum of (three-dimensional) Gaussians, given the choice of viewing angles, and using an MGE model for the PSF, we could then deconvolve and deproject the MGE model uniquely and analytically.

The procedure was performed stepwise. We began fitting the wide field $V$-band image. We then subtracted the outer gaussian components from the high resolution WFPC2 image, and fitted the residual image (central $15^{\prime \prime}$ ). In this step, we had to exclude the innermost 0.4 , to avoid convergence problems probably due to the slightly asymmetric central feature (Fig. 3). We finally fitted the very central arcsecond. Gathering these three parts, we thus obtained a 15 Gaussian components model, with the same center and PA, the parameters of which are given in Table 1. The goodness of the fit is illustrated in Fig. 5.

\subsection{Two-integral models}

We then made use of the Hunter \& Qian (1993) formalism to build the two-integral distribution function of the galaxy using the best fit value for the mass-to-light ratio of $\Upsilon_{V}=6.6 M_{\odot} / L_{\odot}$ found from simple Jeans models. We used a default value of $i=90^{\circ}$, which produced a marginally better fit.

The DF is divided into two parts, which are respectively even and odd in $L_{z}$. The even part $\left(f_{\text {even }}\right)$ is uniquely determined by the input MGE mass model. This involves the calculation of a path integral in the complex plane, as described by Hunter \& Qian (1993). The odd part of the distribution function $\left(f_{\text {odd }}\right)$ is then parametrized, and adjusted to fit the kinematical data. We chose the parametrization proposed by van der Marel et al. (1994), and modified it to account for the CRC. The original parametrization corresponds to Eq. (1), and we additionally allowed $\eta$ to be function of $E_{\mathrm{p}}=E / E_{\max }$ (Fig. 6). The analytical 

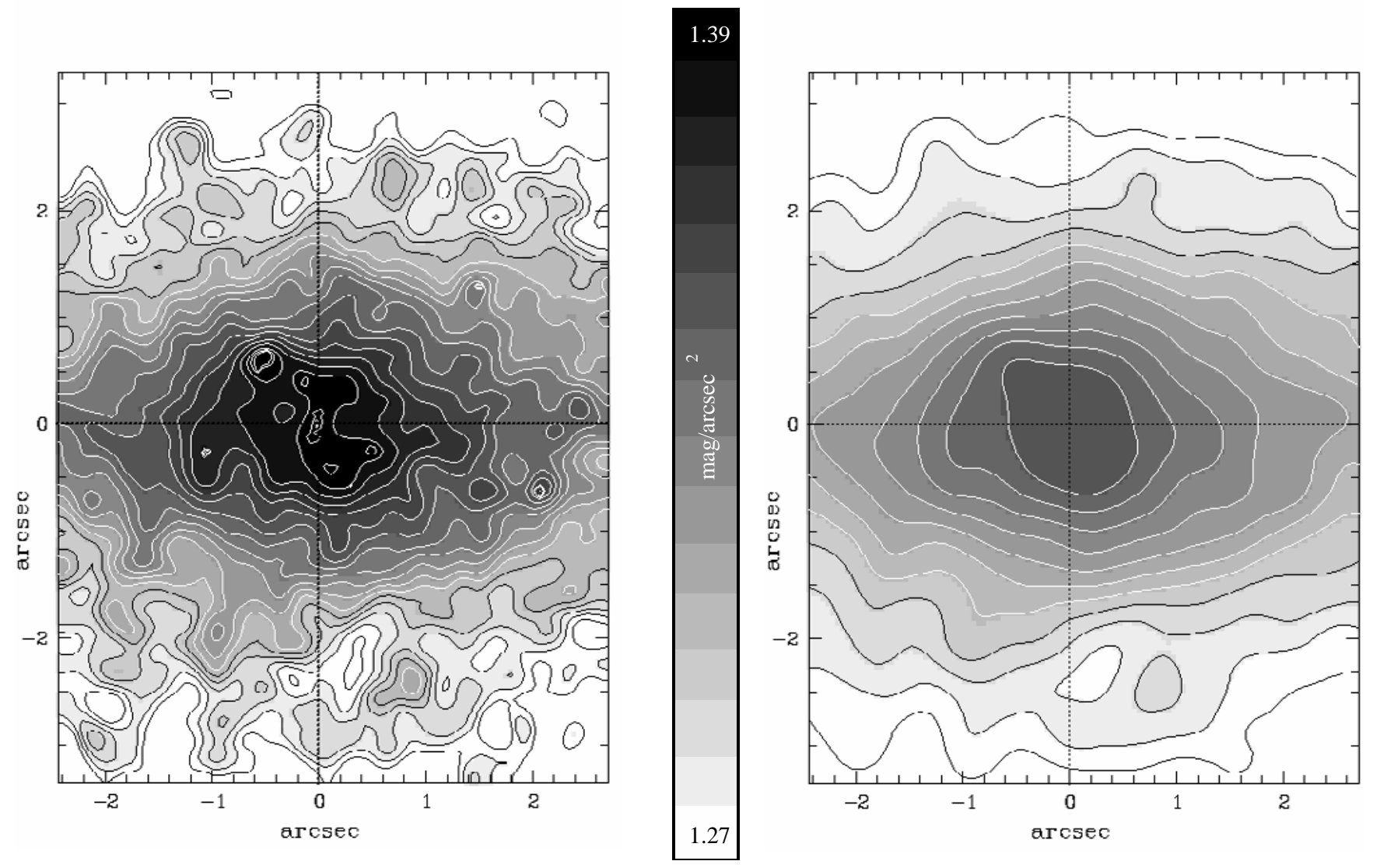

Fig. 4. $V-I$ map (WFPC2, F555W and F814W filters). Left: convolved with a gaussian of $\sigma=0$ '. 1 . Right: convolved with the PSF of the OASIS data $(0.51 F W H M)$. Contour separation is 0.005 mag. A $y$-axis elongated central structure can be observed at HST resolution (see also Fig. 3).

form of this $\eta$ function is the same as the one in Eq. (2), with an additional variable change. We can adjust the energy $E_{\text {crit }}$ above which the stars begin to counter-rotate, as well as the smoothness of the transition ( $a=\infty$ : abrupt transition, $a=1$ : smooth transition, see Fig. 6).

$$
\begin{aligned}
f_{\text {odd }}\left(E, L_{z}\right) & =(2 \eta-1) h_{\alpha}\left(L_{z} / L_{z, \max }\right) \cdot f_{\text {even }}\left(E, L_{z}\right) \\
h_{\alpha}(x) & = \begin{cases}\tanh (\alpha x / 2) / \tanh (\alpha / 2) & (\alpha>0) \\
x & (\alpha=0) \\
(2 / \alpha) \operatorname{arctanh}[x \tanh (\alpha / 2)] & (\alpha<0)\end{cases}
\end{aligned}
$$

The best fit model reproduces the BSG94 velocity profiles reasonably well (Fig. 7) with values of $\alpha$ ranging from 8 outside $10^{\prime \prime}$ to -2 in the central part. The higher resolution OASIS (Figs. 8 and 1), and STIS (Fig. 2) velocity measurements, revealing the counter-rotating core, are also well fitted by this two-integral model. The best fitting model uses a core with a diameter of 1 .' 1 ( $\left.E_{\text {crit }}=0.62\right)$, and an abrupt transition $(a=100$ i.e. almost all stars having $E_{\mathrm{p}}>E_{\text {crit }}$ are counter-rotating, see bold line in Fig. 6). A rough estimate of the CRC mass can be made by selecting stars counter-rotating in the central part. This is performed by integrating the DF weighted by a function which is 0 for $E<E_{\text {crit }}$ and $L_{z}>0$ and 1 otherwise. The total mass of NGC 4621, which is given by the mass-to-light ratio and the deprojected MGE-model is $1.78 \times 10^{11} M_{\odot}$. The total mass of the $\mathrm{CRC}$ is $2.13 \times 10^{8} M_{\odot}$, yielding a mass fraction of $0.12 \%$.

The dispersion profiles are well reproduced by the model outside the central few arcseconds. The central values of the dispersion predicted by the models are however systematically too low compared to the BSG94 observations. This is confirmed by the OASIS and STIS dispersion values: the model predicts a central dispersion of $\sim 220 \mathrm{~km} \mathrm{~s}^{-1}$, to be compared with the central observed STIS dispersion of $320 \pm 27 \mathrm{~km} \mathrm{~s}^{-1}$. We were finally unable to fit the higher order moments, even at large radii. The $h_{3}$ values predicted by the model are thus systematically too high, by a factor of almost two. This discrepancy could not be solved even by changing the parameters of the odd part of the DF.

These two discrepancies do indicate that we need a more general dynamical model for NGC 4621. First, we should remove the constraint imposed by the two-integral model by allowing a third integral of motion. There may then still be the need for an additional central dark mass to explain the observed dispersion values. Such a model will be examined in a forthcoming paper (Wernli et al., in preparation).

\section{Discussion and conclusion}

In this paper, we report the discovery of a $\sim 60$ pc diameter counter-rotating core using new OASIS integral field 

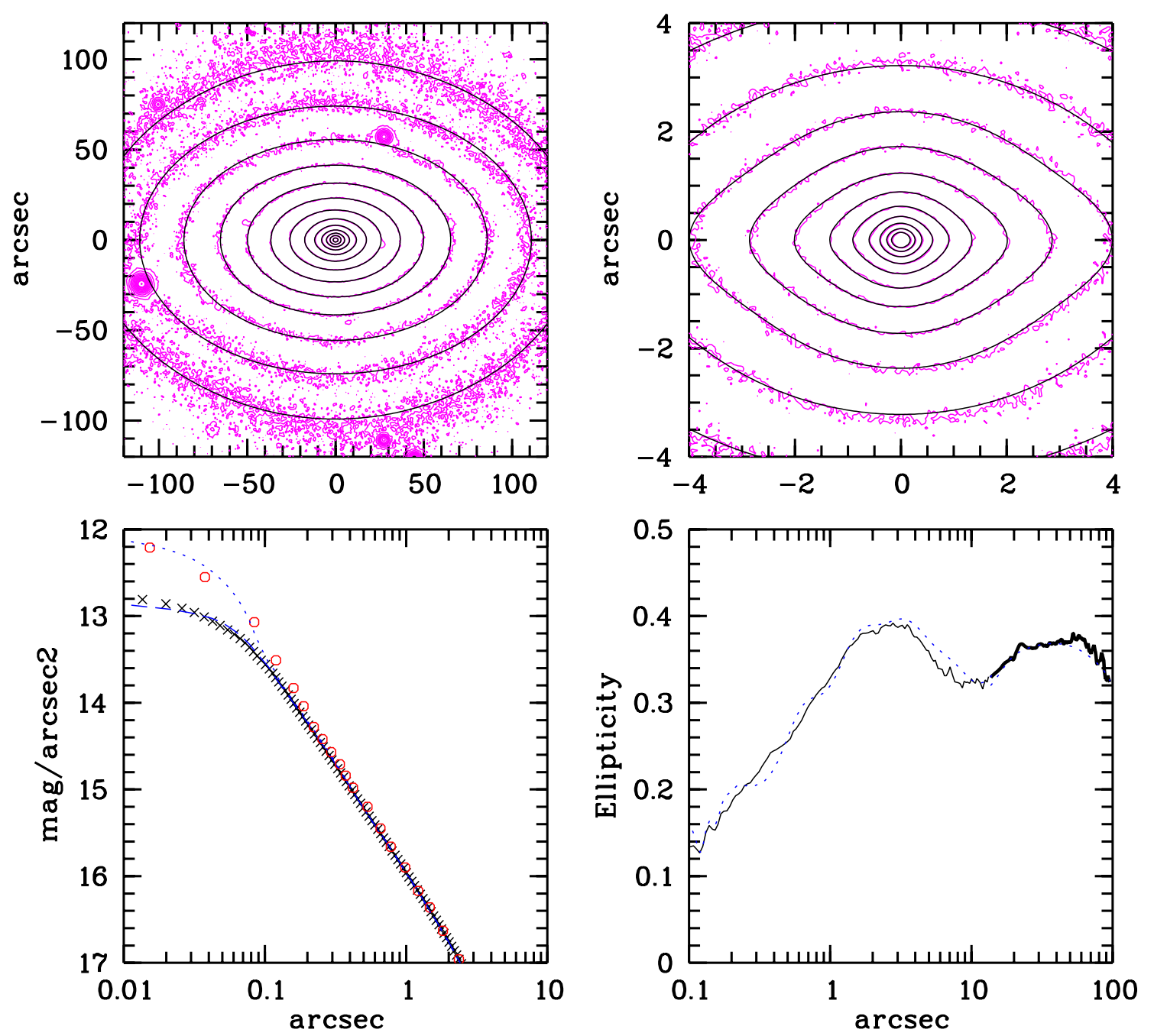

Fig. 5. Top panels: multi Gaussian Expansion fit (thick contours) of NGC 4621 superimposed on the $V$ band isophotes (thin contours). Top left: OHP $V$-band photometry. Top right : HST/WFPC2 F555W band image (isophote step of $0.4 \mathrm{mag} / \operatorname{arcsec}^{2}$ ). Notice the nuclear disc in the HST data. Bottom left panel: NGC 4621 light profiles along $r^{2}=x^{2} / a^{2}+y^{2} / b^{2}$, where $a$ and $b$ are the semi major and minor axes of fitted ellipse respectively. Crosses correspond to the WFPC2 image, circles to the deconvolved WFPC1 data from Byun et al. (1996). The dashed and dotted curves correspond to the convolved and deconvolved MGE models respectively. Bottom right panel: ellipticity profile, with Michard's (solid bold line) and WFPC2 (solid hairline) data, along with the MGE model (dotted line).

spectroscopy, a result confirmed with archival STIS long-slit data. A structure at this scale has never been observed before, except in M 31, which exhibits a 20 pc co-rotating decoupled core (Bacon et al. 2001b and references therein). Typical sizes of kinematically decoupled cores are at the order of $1 \mathrm{kpc}$ (See e.g. the sample of Carollo et al. 1997). The computed mass fraction of $0.12 \%\left(2.1 \times 10^{8} M_{\odot}\right)$ is on the low side, as compared for instance to the CRC of IC 1459, which is $0.5 \%$ for a core size of $3 \mathrm{kpc}$ (Cappellari et al. 2002), but for which the estimation is of higher accuracy. As a comparison, the black hole mass from the $\sigma-M_{\bullet}$ relation for IC 1459 is $1.1 \times 10^{9} M_{\odot}$ (Merritt \& Ferrarese 2001b) and $2.13 \times 10^{8} M_{\odot}$ for NGC 4621 (Merritt \& Ferrarese 2001a). The mass fraction estimate provided here in the case of NGC 4621 can only be indicative: the DF separation is inaccurate and difficult, and to get a precise result we would need an analysis in integral space.

Both individual OASIS and STIS data sets suggest that the CRC of NGC 4621 is slightly off-centered with respect to the center of the outer isophotes. This offset seems consistent with the weak asymmetry detected in the WFPC2/HST $V$ and $I$ frames and the $V-I$ colour map (Fig. 3). The central structure elongated along the minor axis detected in the $V-I$ colour map represents an increase of $V-I=0.03$ which could either be due to an intrinsic stellar population gradient, or to dust. In the latter case, this would correspond to $A_{V}=0.06$ (assuming $R_{V}=3.1$ and $R_{I}=1.86$ from models of the Galaxy, Rieke \& Lebofsky 1985). Note that we do not detect any high frequency structure in the individual WFPC2 $V$ and $I$ frames.

The two-integral models reasonably reproduce the observed velocity profiles in the outer parts, as well as in the region of the CRC (OASIS and STIS). These axisymmetric models do obviously not take into account the observed offcentering. The predicted central value of the velocity dispersion is significantly too low by $50 \mathrm{~km} \mathrm{~s}^{-1}$. We were also unable to simultaneously fit higher order Gauss-Hermite moments: e.g. the predicted $h_{3}$ is systematically too high (see Fig. 7). 


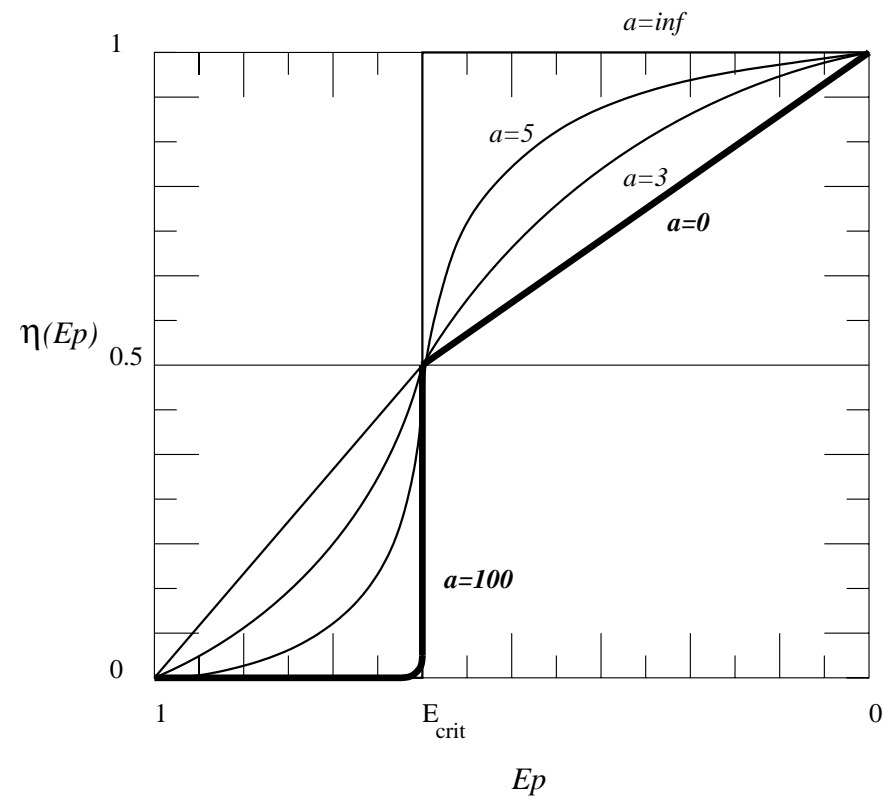

Fig. 6. Parametrization of $\eta$. $E_{\mathrm{p}}$ is the normalized energy $E_{\mathrm{p}}=E / E_{\max }$. $E_{\text {crit }}$ is the energy at which the counter-rotation starts, e.g. when $\eta<$ $0.5, f_{\text {odd }}<0$ (see Eq. (1)). The bold line corresponds to the best-fitting model.

This demonstrates that we need more general dynamical models in order to properly fit the observed kinematics. We are therefore in the process of constructing three-integral Schwarzschild models, with the possibility to include a central dark mass. This may solve the discrepancies mentioned above.

If one assumes a merger-scenario for the CRC's origin, the off-centering, if confirmed, could be either due to the fact that the merging process is still on-going, or may be the result of a stable mode (see e.g. M 31 in Bacon et al. 2001b). The short dynamical timescale at the radius of the CRC (1 Myr) seems to favour the latter hypothesis. Further discussions regarding this issue must however wait for additional spectroscopy. A detailed study at high spatial resolution of the stellar populations in the central arcsecond would certainly help in this context. Existing high resolution STIS spectra at bluer wavelengths can be used for this purpose.

Stellar kinematics at HST resolution is available for only a handful of early-type galaxies. M 31 is one of the rare examples where it is possible to have sufficient spatial resolution to measure kinematical features at parsec scales, such as its $20 \mathrm{pc}$ nucleus (Bacon et al. 2001b and references therein). The OASIS data presented in this paper demonstrate that it is possible to study substructures with a characteristic size of $60 \mathrm{pc}$ in galaxies at the distance of the Virgo cluster. The presence of the CRC in NGC 4621 raises new questions about the dynamical status of the centers of early-type galaxies. What is the fraction of early-type galaxies that have such substructures? Two-dimensional spectroscopy at high spatial resolution is clearly needed to simultaneously study the dynamical and chemical contents of these cores, and to eventually understand their origin.

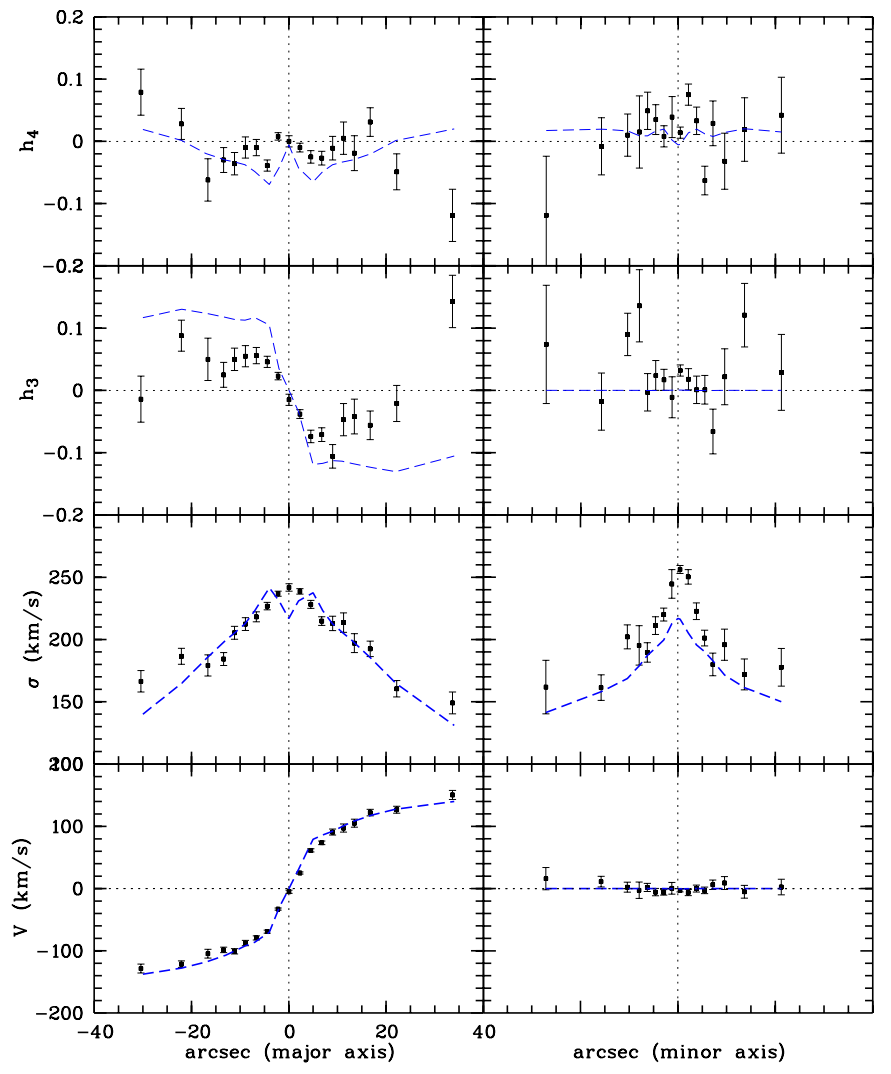

Fig. 7. Best fit two-integral DF model. Left: major axis. Right: Minor axis. Inclination is 90 degrees, no black hole and $\Upsilon_{V}=6.06$. Points are the BSG94 data, and the curve is the model. The fit is reasonable but indicates the need for a black hole (low central $\sigma$ ) and maybe a third integral (overestimated $h_{3}$ ).

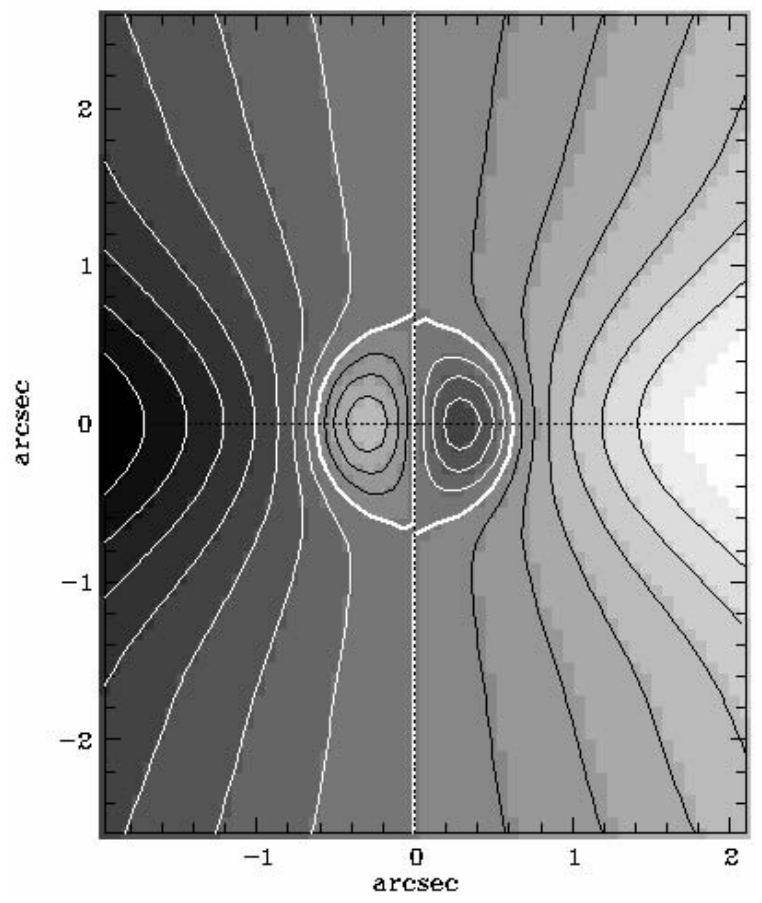

Fig. 8. Best fit two-integral DF model for the comparison with OASIS. Only the velocity map is shown: central dispersion values are too low due to the absense of a central dark mass. Grey levels range from -50 to $+50 \mathrm{~km} \mathrm{~s}^{-1}$. 
Acknowledgements. We would like to thank Raymond Michard and Ralf Bender for the data they provided, Tim de Zeeuw for a careful reading of the manuscript and his suggestions, and the referee Dr M. Carollo for her thorough and constructive report.

\section{References}

Bacon, R., Emsellem, E., Copin, Y., \& Monnet, G. 2000, in Imaging the Universe in Three Dimensions, ed. W. van Breugel, \& J. Bland-Ilawthorn, ASP Conf. Ser., 195, 173

Bacon, R., Copin, Y., Monnet, G., et al. 2001a, MNRAS, 326, 23

Bacon, R., Emsellem, E., Combes, F., et al. 2001b, A\&A, 371, 409

Bender, R. 1988, A\&A, 202, L5

Bender, R., Saglia, R. P., \& Gerhard, O. E. 1994, MNRAS, 269, 785, BSG94

Bender, R. 1990, A\&A, 229, 441

Byun, Y., Grillmair, C. J., Faber, S. M., et al. 1996, AJ, 111, 1889

Cappellari, M., Verolme, E. K., van der Marel, R. P., et al. 2002 [astro-ph/0202155]

Carollo, C. M., Franx, M., Illingworth, G. D., \& Forbes, D. A. 1997, AJ, 481, 710

Cole, S., Aragon-Salamanca, A., Frenk, C. S., Navarro, J. F., \& Zepf, S. E. 1994, MNRAS, 271, 781

Copin, Y. 2000, Dynamique des galaxies de type précoce: observations 3D et modélisations, Ph.D. Thesis, École Normale Supérieure de Lyon van der Marel, R. P., Evans, N. W., Rix, H.-W., White, S. D. M., \& de Zeeuw, T. 1994, MNRAS, 271, 99

Emsellem, E., Monnet, G., \& Bacon, R. 1994, A\&A, 285, 723

Faber, S. M., Tremaine, S., Ajhar, E. A., et al. 1997, AJ, 114, 1771

Gebhard, K, Richstone, D., Ajhar, E. A., et al. 1996, AJ, 112, 105

Goudfrooij, P. 1998, STIS Instrument Sci. Rep., 98-29

Hunter, C., \& Qian, E. 1993, MNRAS, 262, 401

Idiart, T. P., Michard, R., \& de Freitas Pacheco, J. A. 2002, A\&A, 383, 30

Lauer, T. R., Ajhar, E. A., Byun, Y.-I., et al. 1995, AJ, 110, 2622

Merritt, D., \& Ferrarese, L. 2001a, MNRAS, 320, L30

Merritt, D., \& Ferrarese, L. 2001b, ApJ, 547, 140

Moellenhoff, C., \& Bender, R. 1989, A\&A, 214, 61

Monnet, G., Bacon, R., \& Emsellem, E. 1992, A\&A, 253, 366

Rieke, G. H., \& Lebofsky, M. J. 1985, ApJ, 288, 618

Rigaut, F., Salmon, D., Arsenault, R., et al. 1998, PASP, 110, 152

Sil'chenko, O. K. 1997, Astron. Rep., 41, 567

Tonry, J. L., Dressler, A., Blakeslee, J. P., et al. 2001, APJ, 546, 681

Verolme, E. K., Cappellari, M., Copin, Y., et al. 2002, MNRAS, in press

Wagner, S. J., Bender, R., \& Moellenhoff, C. 1988, A\&A, 195, 5

Wagner, S. J. 1990, Dynamics and Interactions of Galaxies, 244-248, Wielen, R. (Springer Verlag)

de Zeeuw, P. T., Bureau, M., Emsellem, E., et al. 2002, MNRAS, 329, 513

de Zeeuw, P. T., \& Franx, M. 1991, ARA\&A, 29, 239 